\title{
Multiphysics Modelling of the Fabrication and Operation of a Micro-Pellistor Device
}

\author{
Ferenc Biró $^{1,2}$, Zoltán Hajnal ${ }^{1, *}$, Andrea Edit Pap ${ }^{1}$, István Bársony ${ }^{1}$ \\ ${ }^{1}$ MEMS Laboratory, Institute of Technical Physics and Materials Science, \\ Research Centre for Natural Sciences, Hungarian Academy of Sciences, \\ Konkoly Thege Miklós út 29-33, H-1121 Budapest, Hungary \\ ${ }^{2}$ University of Pannonia, Egyetem u. 10, H-8200 Veszprém, Hungary \\ *corresponding author, e-mail: <hajnal.zoltan@ttk.mta.hu>
}

\begin{abstract}
Downsizing efforts in gas-sensing applications lead to ever smaller active elements. Integration with data processing circuitry requires the use of CMOS compatible fabrication technology, autonomous operation poses limits on energy consumption of the elements, whereas reliable catalytic detection often needs high temperatures that may otherwise be constrained by safety considerations. Under these conditions, development of active sensor elements proves to be a growing challenge for design and fabrication.

In this work we present a step-by-step study on a $\approx 500 \mu \mathrm{m}$ diameter thermally isolated membrane element of a gas detecting microsensor device. Sensitivity is based on high temperature $\left(\approx 3-400{ }^{\circ} \mathrm{C}\right)$ catalytic activity of a porous pellistor deposited on a multilayer $\mathrm{SiO}_{2} / \mathrm{SiN}_{\mathrm{x}}$ filament heated - membrane that has to be durable enough for several thousand hours of operation, and as thin as possible to reduce heat conduction to the substrate. $\mathrm{SiO}_{2}$ membranes tend to show high residual stress that can be significantly reduced by "sandwiching" with $\mathrm{SiN}_{\mathrm{x}}$.

We have used COMSOL Multiphysics ${ }^{\circledR} 4.3 a$ [1] to assist the initial product design, and evaluation of operational constrains of the multi-layer thin film. The first part involved systematic thermo-mechanical iterations, while the latter consisted of a combination of gradual static thermo-electro-mechanical simulation steps.

As shown by simulating the steps of the deposition process in this work, the right combination of different techniques produces a stable 4-layer membrane with only a sub-micron deformation, and tolerable residual stresses after membrane forming (substrate removal) and during operation. Also, the pellistor filament heating power should be minimized and still reach the operating temperature of the catalyst hotspot. This design, supported by our model calculation was used to realize the device with targeted characteristics. The structure endures the distortion and thermal expansion and contraction during the heating cycles, whereas low power operation widens the range of possible applications.
\end{abstract}

\section{Introduction}

Multilayer membrane structures are becoming more and more ubiquitous as building elements in commercial MEMS based devices. Their composition and dimensions can be diverse, depending on the intended function and operating conditions of the device. Micro-heaters are also widely utilized MEMS elements in catalytic micropellistors and semiconductor gas sensors, or applied as micro sized infrared (IR) sources. These devices have to be able to operate between $150-600{ }^{\circ} \mathrm{C}$ depending on their functionality under both static and dynamic conditions, often with tight limits on power consumption and high reliability requirements.

Electronics integration assumes the use of CMOS compatible fabrication technologies, which - in practice confine the choice of membrane materials to oxides and nitrides of the wafer base material, silicon. However, their thermal coefficients are quite different from that of silicon. Mechanical stability and several thousand hours of desired operating time require the reduction of residual stress of the membranes, either in perforated or full membrane type gas sensors. Composite membranes are prepared and post processed - usually at elevated temperatures - in multiple cycles by thermal oxidation, LPCVD, and/or other deposition techniques. These processes determine the final electrical and mechanical properties of the device, thus they must be tailored for the optimal operating temperature and lifetime.

Thin film structures can be regarded stress free if the residual mechanical stresses are below $100 \mathrm{MPa}$ [2]. There are two possible ways to reduce the residual stress in these structures. In the application of nonstoichiometric $\mathrm{SiN}_{\mathrm{x}}$ the residual stress could be controlled by changing the silicon-nitrogen ratio in the deposited film. Nevertheless, $\mathrm{Si}$ rich $\mathrm{SiN}_{\mathrm{x}}$ films offer reduced thermal isolation.

The alternative is using a stacked composition of $\mathrm{SiO}_{2}-\mathrm{Si}_{3} \mathrm{~N}_{4}$ layers. Due to the differences in the magnitudes and the opposite sign of the residual stresses in $\mathrm{SiO}_{2}$ and $\mathrm{Si}_{3} \mathrm{~N}_{4}$, it is possible to tune a composite membrane to be almost stress-free.

Further, to increase power efficiency of the device, membranes need to be bad thermal conductors in order to minimize heat dissipation. Since heat conductivity in the freestanding part of the membrane is strongly depending on its thickness and composition, and the effect of the metallic filament must also be taken into account, accurate simulation of the thermal properties of the layer structures can be crucial during the design phase. As these devices operate either in ambient conditions, or in other gaseous medium, heat dissipation through diffusion and convection cannot be neglected either. Radiation loss may also reduce power efficiency.

In the following we present the basic design criteria of the active element of our micro-hotplate gas sensor device, describe the details of the multi-stage simulation 
of various steps of the membrane fabrication, concentrating on the thermo-mechanical performance. The simulation is completed then with a parametric study of the thermo-electro-mechanical behaviour of the whole device element. Validation of the models is provided by electrical, thermal, and optical interference measurements on several fabricated prototypes of the device.

\section{Design and fabrication}

The targeted sensor element, the micro-pellistor is to be realized through CMOS compatible technology. Thus the micro-heaters are formed on silicon wafers by multiple thin film growth and deposition techniques. First a $300 \mathrm{~nm} \mathrm{SiO}$ layer is grown thermally at $1200{ }^{\circ} \mathrm{C}$, then

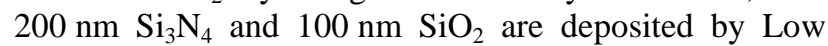
Pressure Chemical Vapour Deposition (LPCVD) and atmospheric Chemical Vapour Deposition (CVD) at $800{ }^{\circ} \mathrm{C}$ and $430{ }^{\circ} \mathrm{C}$, respectively. Between every deposition step, the sample is moved to a different chamber, thus cooled down to room temperature (RT). A heating filament (of $4 \mu \mathrm{m}$ wire-width covering $\approx 100 \times 100 \mu \mathrm{m}^{2}$ surface area) is then formed: using a masked deposition technique first a thin $(<100 \mathrm{~nm}) \mathrm{TiO}_{2}$ adhesion layer, and then $\mathrm{Pt}$ metal is sputtered to achieve a total of $\approx 270 \mathrm{~nm}$ thickness.

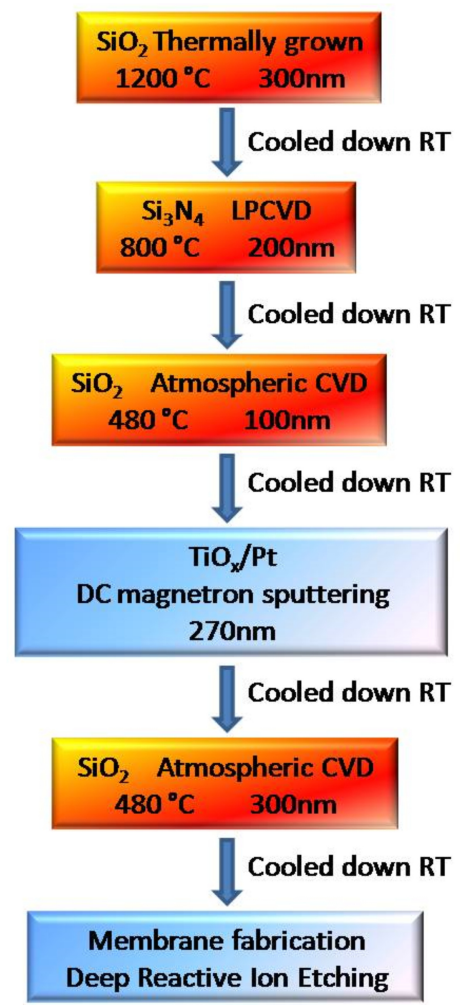

Figure 1: Process flow chart of micro-pellistor fabrication. Red boxes signalize high temperature treatment, blue ones close to room temperature (RT).

To provide chemical and electrical isolation, an additional $300 \mathrm{~nm} \mathrm{SiO}_{2}$ layer is deposited by CVD after removal of the sputtering mask. A freestanding membrane is produced by Deep Reactive Ion Etching (DRIE) from the backside of the wafer that "drills" a 300-320 $\mu \mathrm{m}$ diameter cylindrical cavity centred under the filament. These basic processing steps are summarized in Figure 1. Further details about the device fabrication and the experimentally tested structures had been described elsewhere [3].

While wafer processing enables efficient mass production, the physical behaviour of the micro-heaters can be simulated for an individual element. Starting with a $400 \times 400 \mu \mathrm{m}^{2}$ slab of a $370 \mu \mathrm{m}$ thick pure silicon wafer, the oxide-nitride-oxide membrane "sandwich" was constructed using the model builder in COMSOL. Lacking specific empirical material parameters of the individual layer component materials, the default properties of the Materials Library of the MEMS module for $\mathrm{Si}, \mathrm{Pt}, \mathrm{SiO}_{2}$, and $\mathrm{Si}_{3} \mathrm{~N}_{4}$ have been used. The thin adhesion layer of $\mathrm{TiO}_{2}$ was ignored in simulations to simplify the layer and mesh construction. Figure 2 shows the overview of the structure.

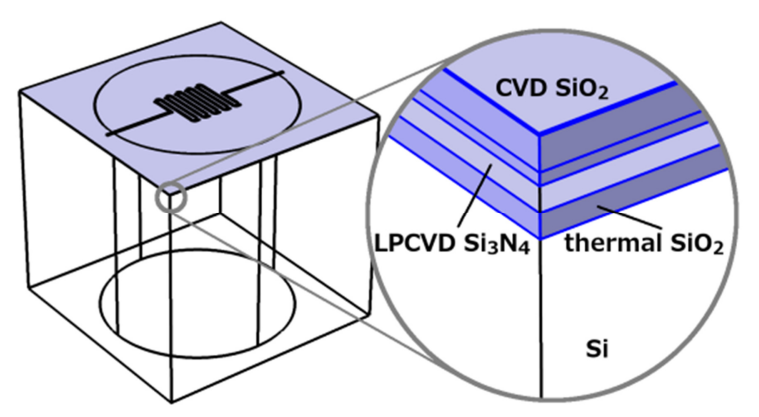

Figure 2: Overview of the model geometry with the layer composition of the membrane in the blow-up.

An appropriate mesh must be added to the geometry model, over which the coupled equations of the simulations are calculated. In our case, the thin film components require special attention, as the automated meshing would produce enormous amounts of mesh elements, adjusting sizes of mesh tetrahedra to the thickness of the films. We need to accurately simulate temperature dependent transfer processes of current generated heat and its dissipation where its gradient is expected to change most rapidly.

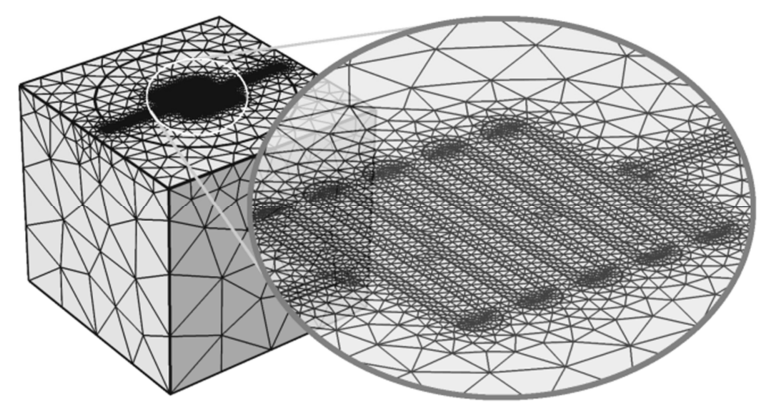

Figure 3: The structure of the adapted mesh (see text). Blow-up: refinement around the Pt filament. 
Thus a reasonable way is to start from a triangular mesh on one lateral face of the filament, with the elements' lower $(500 \mathrm{~nm})$ and upper $(2 \mu \mathrm{m})$ size limits commensurate with the layer thickness. A detail of the mesh is shown in Figure 3. Letting the triangular mesh run over the whole nitride surface, gradually expanding toward the edges, then sweeping this triangular distribution vertically through the membrane (allowing 2 divisions of each sub-layer), and finishing the bulk part with a tetrahedral mesh, results in $\approx 65000$ elements. This pushes the model to the computational limits affordable on a multicore workstation with 16GB memory. The final thermo-electro-mechanical simulation involves solutions for over $10^{6}$ degrees of freedom.

To account for the fabrication recipe stages, each step is reproduced as a static "thermal stress" calculation in COMSOL. In the first 5 steps - as in Figure 1 - new layers are added on top of the structure one-by-one, while the layers below inherit the residual stresses from the previous step. The reference temperatures are set to the processing temperature of the given step, and the system is relaxed then to room temperature (RT). As it can be seen in Figure 4, local residual stress is strongly material and processing temperature dependent. In accordance with literature values [4], the first (bottom-most) thermal oxide layer sustains the most residual stress $(\approx 0.3 \mathrm{GPa})$, which is relieved by the subsequent nitride and oxide layers.

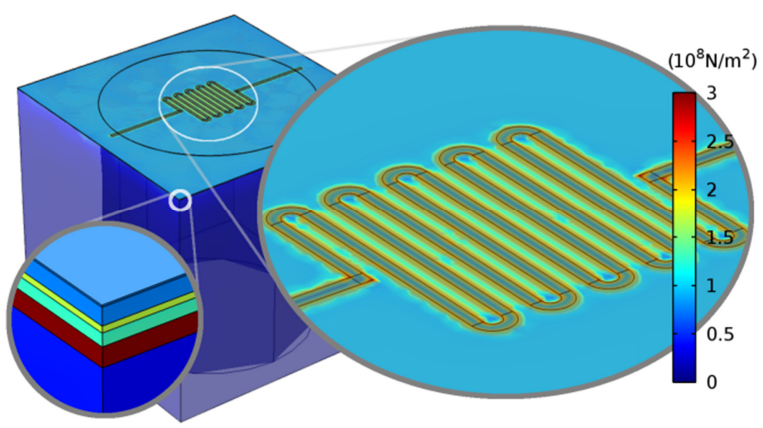

Figure 4: Von Mises stress after the five stages of simulated membrane formation.

\section{Physics of device operation}

Membrane formation and the simulation of operating conditions are finally unified in one parametric simulation stage. The necessary initial conditions are defined by the residual stresses from the last deposition step, and removal of the cylindrical middle part of the substrate. In this concluding stage a current is driven through the $\mathrm{Pt}$ filament. Besides other thermal properties, the propagation of the generated heat is calculated simultaneously with the thermal expansion of the membrane until a steady state is achieved for given current values, by taking the temperature dependence of the electrical conductivity also into account.

There are three ways of heat dissipation in this model system. The most important one is conduction through the substrate itself, which is modelled by setting the bottom plane of the sample to room temperature. Less pronounced - but certainly not negligible at higher temperatures - is the convective heat transfer to the surrounding medium. In our case normal ambient air was chosen, with the dissipation parameters as provided by COMSOL, using the whole surface of the sample. The least contribution at the typical operating temperatures is expected from radiation. For the simulations we chose a uniform 0.8 as radiative coefficient, and room temperature for the surroundings.

\begin{tabular}{|r|r|r|}
\hline current $[\mathbf{A}]$ & max. $\mathbf{T}[\mathbf{K}]$ & $\boldsymbol{\Delta} \mathbf{z}[\boldsymbol{\mu \mathbf { m }}]$ \\
\hline 0.001 & 297 & -5.0 \\
\hline 0.002 & 311 & -4.9 \\
\hline 0.003 & 335 & -4.7 \\
\hline 0.004 & 375 & -4.3 \\
\hline 0.005 & 441 & -3.7 \\
\hline 0.006 & 553 & -2.6 \\
\hline 0.007 & 749 & -0.6 \\
\hline 0.008 & 1049 & 2.7 \\
\hline 0.009 & 1382 & 6.8 \\
\hline 0.010 & 1638 & 10.5 \\
\hline
\end{tabular}

Table 1: Maximal temperature and vertical displacement values as function of the pellistor heating current.

Maximal values are listed in Table 1. Figure 5 shows the resulting temperature and stress distributions of the device at two different current values. Vertical displacement of the membrane is exaggerated to 5 times of the actual, and the original contour lines of the filament are left in place to emphasize membrane distortion (otherwise barely visible).
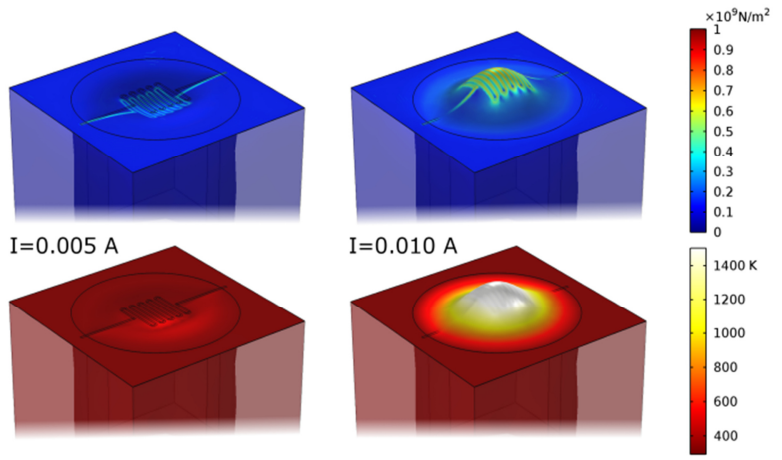

Figure 5: Von Mises stress (top) and temperature (bottom) distribution of the simulated micro-pellistor driven by $5 \mathrm{~mA}$ (left) and $10 \mathrm{~mA}$ (right).

\section{Comparison with electrical measurements}

To assess the electrical properties of the fabricated devices measurements have been carried out using a Keithley 2400 SourceMeter $^{\circledR}$, using the DC current generator for filament heating. Cold resistance of the micro-hotplate (measured at $100 \mu \mathrm{A}$ ) was $181.2 \Omega$ exceeding the $118 \Omega$ of the same in the COMSOL model. As it can be clearly seen in Figure 6, under operating 
conditions the measured resistance rises with increasing temperature more slowly than the simulated one.

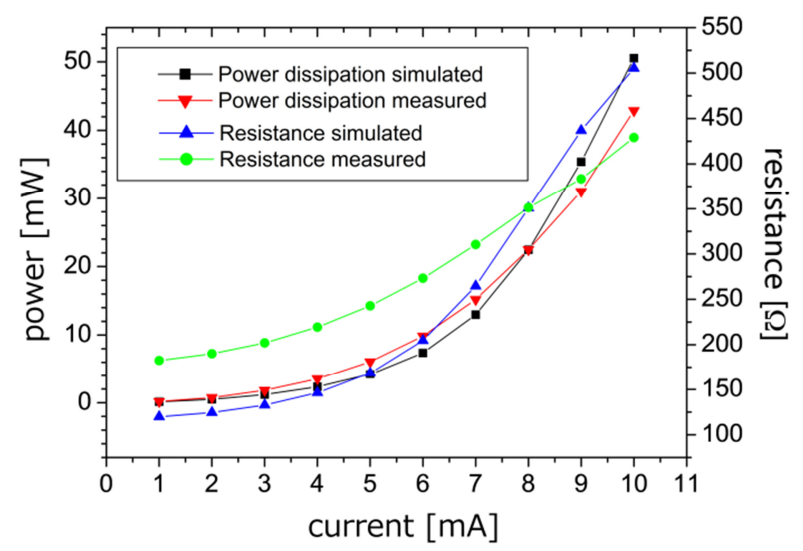

Figure 6: Resistance and power dissipation of the microhotplate in measurements and simulation.

The difference in the "cold" value can mostly be attributed to the larger cross-section of the filament in the simulation (as the $\mathrm{TiO}_{2}$ sub-layer is ignored). As the $\mathrm{Pt}$ filament is deposited by magnetron sputtering it may contain more defects and voids, which may also alter its temperature coefficient of resistance (TCR). In our device, the measured TCR value is $\alpha_{0}=3.1 \cdot 10^{-3}$, whereas the ideal $\left(3.3 \cdot 10^{-3}\right)$ is used in the COMSOL material properties definition.

\section{Comparison with thermal measurements}

One has to find a suitable indirect method to measure the temperature of the tiny micro-membranes with acceptable accuracy.

\begin{tabular}{|l|r|}
\hline \multicolumn{1}{|l|}{ Salt } & $\mathbf{T}_{\text {melting }}\left[{ }^{\mathbf{}} \mathbf{C}\right]$ \\
\hline $\mathrm{AgNO}_{3}$ & 212 \\
\hline $\mathrm{KNO}_{3}$ & 334 \\
\hline $\mathrm{Pb}\left(\mathrm{NO}_{3}\right)_{2}$ & 470 \\
\hline $\mathrm{V}_{2} \mathrm{O}_{5}$ & 690 \\
\hline $\mathrm{Na}_{2} \mathrm{SO}_{4}$ & 884 \\
\hline
\end{tabular}

Table 2: Transition temperatures of the inorganic compounds, used in micro-melting point measurements.

We have applied a micro-melting point calibration technique to determine the temperature / heating power relationship of our micro-hotplate. Interior areas of the filaments were coated by glycerol suspended inorganic compounds, known to exhibit sharp solid-liquid phase transition at their melting point (see Table 2). While slowly increasing the filament current, the phase transition was observed under optical microscope. Every measurement was repeated on five different samples and the measured power values were averaged to obtain the temperature of the hotplate. Figure 7 shows the average and maximum temperatures of the membrane surfaces from the COMSOL simulation, along with the measured micro-melting points of the 5 salts listed in Table 2. Simulated average temperatures show a fairly good correspondence to the experimentally determined values, while maxima are well above them.

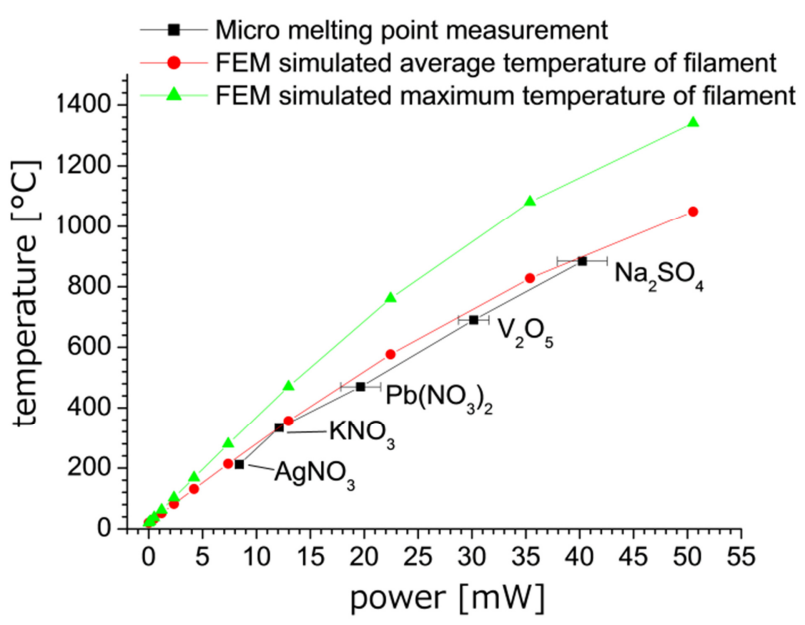

Figure 7: Simulated and measured temperature/heating power relationship of the micro-hotplate.

\section{Thermo-mechanical distortion}

For long-term stability and reliable operation of a device it can be crucial to assess thermal stress related mechanical load on the membrane in the duty cycle. Using a Bruker ContourGT-I Optical Microscope White Light Interference (WLI) based 3D topographic images were recorded at room temperature (Figure 8) as well as heated by currents of $6,8,10 \mathrm{~mA}$ (Figure 10).

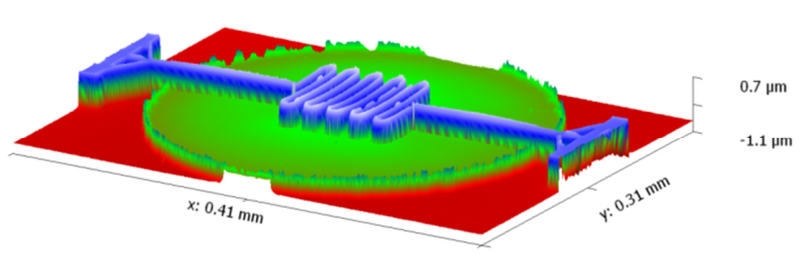

Figure 8: 3D optical microscopy image of a microhotplate at room temperature.

These topographic images are constructed from the interference patterns recorded digitally. In our case, we are dealing with a multilayer structure with several interfaces, all of which contribute somewhat to the interferences. In the $3 \mathrm{D}$ reconstruction, we can clearly identify the structural components of the micro-hotplate, with a certain offset caused by variation of the reflection signal at their boundaries. Observing a cross-section, the morphology of individual elements and even some layer thicknesses can be deduced from the regularities in their appearance. For better understanding, a line profile across the membrane area was plotted in the inset of Figure 9 indicating the path with a black line. At the outer edges of the membrane two peaks occur due to the sharp transition between silicon and cavity (etched out by DRIE to complete the membrane fabrication). Here a rapid change occurs in the intensity of interference lines that the instrument cannot process correctly, so these artefact peaks can be ignored. The level shift between the $\mathrm{Si}$ 
substrate and the membrane is approximately $300 \mathrm{~nm}$ that corresponds to the thickness of the first (thermal) $\mathrm{SiO}_{2}$ layer. The height of the intense peaks in the filament area equals the sum of the layer thicknesses of $\mathrm{Si}_{3} \mathrm{~N}_{4}(200 \mathrm{~nm})$, $\mathrm{SiO}_{2}(100 \mathrm{~nm}), \mathrm{TiO}_{\mathrm{x}} / \mathrm{Pt}(15 / 270 \mathrm{~nm}), \mathrm{SiO}_{2}(300 \mathrm{~nm})$.

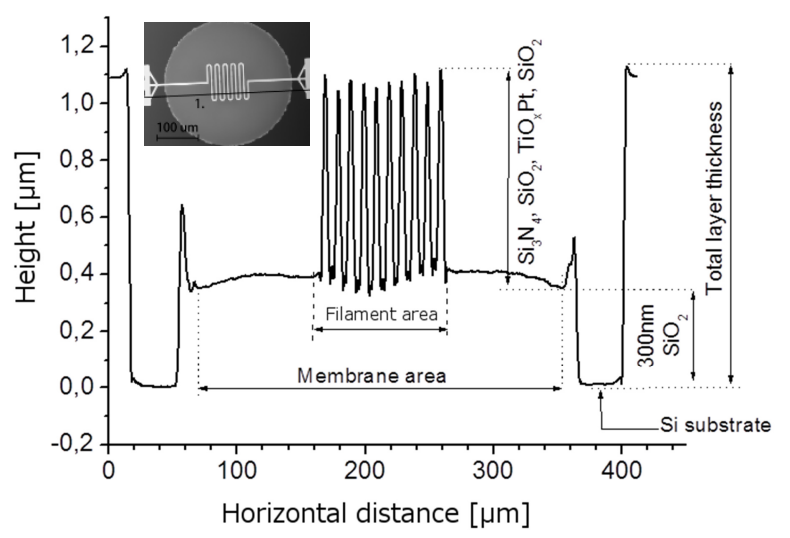

Figure 9: Line profile analysis of a room temperature device showing the components giving rise to the features in the height distribution. Inset: position of the line scan on the membrane.

At room temperature, the interior area of the filament is slightly bent downward, while the outer membrane regions show a slight upward buckling. At higher temperatures not only the filament area, but also the whole membrane increasingly buckles downward, as illustrated by Figure 10.

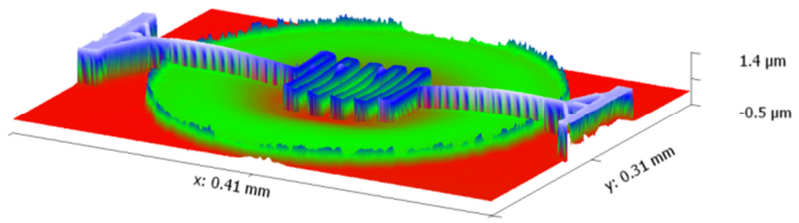

Figure 10: $3 \mathrm{D}$ reconstruction of the heated membrane driven by $10 \mathrm{~mA}$.

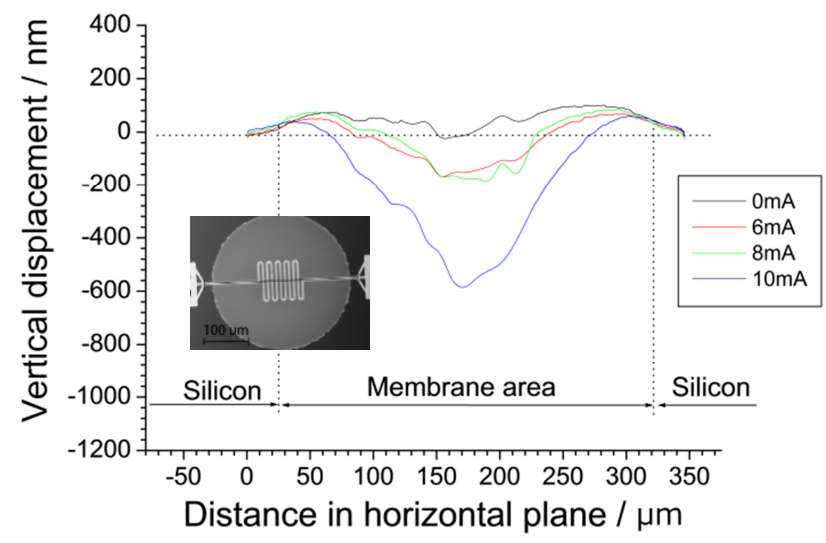

Figure 11: Line profiles at various heating currents corrected for the variation of interference peaks over the filament. Inset: scan line position on the membrane.
Using a combination of multiple line profiles (to subtract the intense level shifting around the filament) a filtering can be carried out to obtain a cross-sectional view of the distorted membrane surfaces at different driving currents. The results of this analysis are summarized in Figure 11.

This observed mechanical behaviour is in contrast to that predicted by the simulations (see Table 1 and Figure 5), both qualitatively and quantitatively. Several attempts were made to fine-tune the geometry parameters of the model, but keeping the given processing recipe of the layer deposition sequence does not allow for an easy remedy to this difference.

However, during the design of the device, many different options for the layer composition had been explored. There is a combination of the deposition sequence, which - besides resulting in comparable thermal behaviour - also exhibits the downward buckling behaviour upon heating described above.

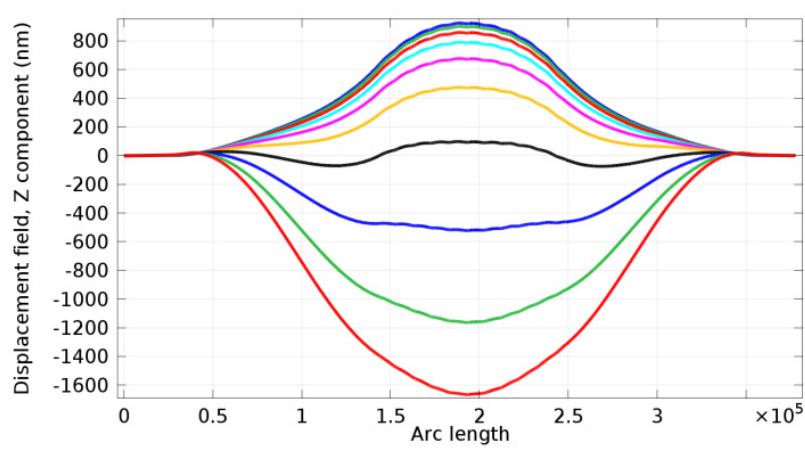

Figure 12: Vertical displacement in an alternative membrane composition (see text).

Such an example is shown in Figure 12. The different line colours correspond to heating currents from 0 to $10 \mathrm{~mA}$, from the top (blue) to the bottom (red). The main difference with this latter structure is that after the thermal oxide layer, deposition of the filament follows immediately, then the nitride, and the other oxide layer.

The "flipping" of the buckling direction is actually representing a reliability hazard, since during operation the bending stress changes sign. It may lead to early fatigue failure, and has to be avoided.

In Figure 11 a saucer shaped line-scan profile is obtained for all loading conditions. It cannot be reproduced by any of the models. In the practical case the apparent buckling along the perimeter of the membrane might be a measurement artefact rather than a real phenomenon, as suggested by the simulations, where no such effect was visible close to the rim of the membrane. It is obvious that the deformation of the thick Si substrate around the membrane cannot reflect a line-scan maximum similar to Figure 11.

We are confident that the processing steps (also confirmed indirectly by the $3 \mathrm{D}$ topography) produce the intended structural composition of the membrane, thus the above-mentioned qualitative agreement can only be 
regarded as coincidental. Nevertheless, as the behaviour can be reproduced under different circumstances, a deeper analysis - and possibly optimization - of the temperature dependent material parameters is expected to show a way worth pursuing. The elastic parameters of the CVD oxide layers under different conditions certainly will have pronounced effects on the final thermo-mechanical simulation stage.

\section{Conclusions}

We constructed a parametrized model of a multilayer micro-pellistor device element, and carried out coupled electro-thermo-mechanical simulation of its operation. The electrical and thermal properties of the model provide a sufficiently close approximation of the device properties, enabling efficient analysis in the device design phase.

For the thermo-mechanical behaviour of the studied class of systems, extra care must be taken. The declaration of the parameters of the base materials - more specifically those of the CVD deposited $\mathrm{SiO}_{2}-$ could, as in the presented case they certainly do, need project specific optimization and more thorough validation.

\section{Acknowledgements}

This work was partially supported by the Hungarian KMR 12-1-2012-0031 and KMR 12-1-2012-0107 projects.

\section{References}

1. http://www.comsol.com/

2. Laconte, J. et al, Micromachined Thin-Film Sensors for SOI-CMOS Co-Integration, Springer (Dordrecht, 2006), pp. 64-65.

3. Biró, F. et al, "Optimisation of low dissipation microhotplates - Thermo-mechanical design and characterization," 19th International Workshop on Thermal Investigations of ICs and Systems (THERMINIC), Berlin, Germany, September 2013, pp. 116-121, doi: 10.1109/THERMINIC.2013.6675223

4. MEMS Clearinghouse, IEEE MEMS Workshop, Florida, US, February 1993, p. 223, http://www.memsnet.org/material/silicondioxidesio2fi $\underline{\mathrm{lm} /}$ 